\title{
Article
}

\section{Encapsidation of different plasmonic gold nanoparticles by the CCMV capsid protein}

\author{
Ana L. Durán-Meza, Martha I. Escamilla-Ruiz, Xochitl F. Segovia-González, Maria V. Villagrana- \\ Escareño, J. Roger Vega-Acosta, Jaime Ruiz-Garcia* \\ Biological Physics Laboratory, Institute of Physics, Universidad Autónoma de San Luis Potosí, Álvaro \\ Obregón 64, San Luis Potosí, S. L. P., 78000 México; analuisa.duranmeza@gmail.com (A.L.D-M); \\ iggy_27@hotmail.com (M.I.E-R); xochitl.guao@gmail.com (X.F.S-G); veronica.villagrana@gmail.com \\ (M.V.V-E); rogerveg@mail.ifisica.uaslp.mx (J.R.V-A) \\ * Correspondence: jaime@mail.ifisica.uaslp.mx; Tel.: 011-52-444-8262362
}

\begin{abstract}
Different types of gold nanoparticles have been synthesized that great potential in medical applications such as medical imaging, bio-analytical sensing and photothermal therapy. However, their stability, polydispersity and biocompatibility are major issues of concern. For example, the synthesis of gold nanorods, obtained through the elongated micelle process, produce them with a high positive surface charge that is cytotoxic. While gold nanoshells are unstable and within a few weeks they decompose due to Ostwald ripening. In this work, we report the self-assembly of the capsid protein of cowpea chlorotic mottle virus (CCMV) around spherical gold nanoparticles, gold nanorods and gold nanoshells to form virus-like particles (VLPs). All gold nanoparticles were synthesized or treated to give them a negative surface charge, so they can interact with the positive $\mathrm{N}$-terminus of the capsid protein leading to the formation of the VLPs. To induce the protein selfassembly around the negative gold nanoparticles, we use different $\mathrm{pH}$ and ionic strength conditions that were determined from the capsid protein phase diagram. The encapsidation with the viral capsid protein confers them better biocompatibility, stability, monodispersity and a new biological substrate on which one can introduce specific ligands towards particular cells, broadening the possibilities of medical application.
\end{abstract}

Keywords: Plasmonic Nanoparticles; Gold Nanoparticles; Gold Nanorods; Gold Nanoshells; CCMV; Virus-Like Particles.

\section{Introduction}

Most viruses protect their genomic material through a protein shell called capsid. The virus capsid has various interesting properties such as monodispersity, stability and, in most cases, the capsid proteins self-assemble around its genomic material spontaneously to form the virion. Furthermore, it has been shown that the capsid protein of some viruses can self-assemble around negative cores that mimic its genomic material [1]. Even more, it has also been shown that under the appropriate conditions, capsid proteins can self-assemble without its genome to form empty capsids [2]. Therefore, the viral capsids could be used as nanocontainers of various synthetic or biological materials. However, in order for the exogenous material to be encapsidated, it has to have similar characteristics as those of the native viral genetic material such as charge and size [3].

Cowpea chlorotic mottle virus (CCMV) is an icosahedral plant virus that infects Vigna Unguiculata [4]. The capsid is made of 180 identical proteins and has a so-called triangulation number, $\mathrm{T}=3$. At $\mathrm{pH}<6$ it has an external diameter of $\sim 28 \mathrm{~nm}$ and an internal diameter of $\sim 21 \mathrm{~nm}$ [5]. At higher $\mathrm{pH}^{\prime}$ s the capsid increase its size as it swells [6]. CCMV was the first icosahedral virus to be disassembled and re-assembled in vitro, when their subunits were put together in an appropriate solution [6], and it has been proved that other similar viruses can also self-assembly in a similar way [7]. In most cases, the self-assembly process happens spontaneously just by mixing together the capsid protein with its genetic material. The interactions that lead to the virion self- 
assembly formation are electrostatic in nature, between the interior part of the capsid protein and its genome; since the genomes in solution are negatively charged and the capsid protein have a positive $\mathrm{N}$-terminus that interacts with the genome[8]. CCMV is easy to amplify [4] and there are well known methods for its disassembly, protein purification and reassembly [6] with and without RNA [7]. Furthermore, the capsid protein has been shown to be biocompatible [9], which makes the CCMV capsid an excellent candidate for its use as a nanocontainer for biological applications.

Remarkably, the purified capsid protein of CCMV, under certain conditions, can re-assembly not only into empty capsids, but also form other structures such as tubes, disks and multi-wall capsids [10,11]. This type of assemblies have been studied under a variety of different conditions of ionic strength (I) and $\mathrm{pH}$ [11], temperature, protein N-terminus deletion effect [12], and protein concentration [8]. It turns out that the isoelectric point (pI) of both the capsid and the CP are of capital importance for the understanding of the CP protein self-assembly, since they are directly related with the CP self-assembly phase diagram; a I vs. $\mathrm{pH}$ phase diagram of the CCMV CP has been constructed [11], which had helped in our understanding of the self-assembly behavior of the CCMV CP in the absence of its genomic material. Various capsid polymorphs have been identified by electron microscopy, as is shown schematically in Figure $1[8,11]$. Where we can find four main regions; corresponding to the formation of icosahedral $\mathrm{T}=3$ capsids, multiwall capsids, tubes and disassembled protein.

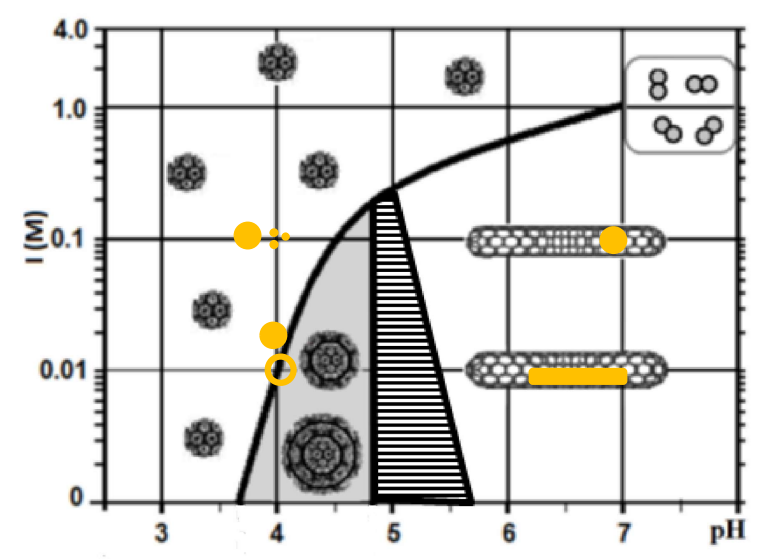

Figure 1. Phase diagram of the CCMV CP self-assembly as a function of $\mathrm{pH}$ and ionic strength. The golden color structures represent each of the different gold nanoparticles; gold nanospheres $(\varnothing \sim 18$ and $\sim 5 \mathrm{~nm}$ in diameter), gold nanoshells $(\varnothing \sim 25 \mathrm{~nm}$ ) and gold nanorods (aspect ratio $\sim 4.1 \mathrm{~nm}$ ). Each structure is placed at the conditions at which the VLP assemblies were formed. Figure was adapted from ref. [8].

The region of icosahedral $\mathrm{T}=3$ corresponds to the formation of empty capsids, identical in size to the native virion. At low $\mathrm{I}$, this region starts from low $\mathrm{pH}$ and ends at $\mathrm{pH} 3.7$, where the isoelectric point of the $\mathrm{CP}$ is located. At high I, this region expands and goes from low $\mathrm{pH}$ to about $\mathrm{pH}$. The multiwall capsid region is delimited at low ionic strength by the isoelectric points of the capsid and the $\mathrm{CP}$, pH 3.7 and 4.8, respectively. In this region, the CCMV capsid and the $\mathrm{CP}$ have opposite charges, therefore the formation of multi-wall capsid structures are driven mainly by electrostatic interactions. As the ionic strength increases or the $\mathrm{pH}$ is lowered, the electrostatic interactions get weaker and one enters the single, empty capsid region. However, the competing interactions, that arise from the $\mathrm{pH}$ and I changes, makes the line transition delimiting the multi-wall capsid and single capsid regions to curve out. On the other hand, the end of the multi-wall capsid region at $\mathrm{pH} 4.8$ is not affected by changes in I and remains constant. It has been proposed that at this $\mathrm{pH}$ value, the protein dimer changes its spontaneous curvature, which destabilizes the formation of pentamers and favors the formation of hexamers; making the transition from multi-wall shells to single and multiwall protein tubes [8]. However, there seems to be a coexistence region between capsids and tubes: The tube region is placed at $\mathrm{pH}$ higher than 5.6 and I lower than $0.5 \mathrm{M}$. The free -mostly dimers- 
protein region is located at $\mathrm{pH}$ greater than 7 and I higher than $0.5 \mathrm{M}$ where there is no attractive interactions to induce the self-assembly of any type of capsid. This diagram help us to choose the appropriate $\mathrm{pH}$ and I to generate virus-like particles (VLPs) for the different gold nanoparticle shapes; the conditions for each assembly are schematically shown as colored structures in Figure 1, corresponding to the shapes of the gold nanoparticles used.

Several groups had use CCMV CP to encapsidate different substances such as negatively charged nanoparticles of $\mathrm{TiO}_{2}$ [13], a negatively charged polymer such as polystyrene sulfonate (PSS) [14], Prussian blue nanoparticles [15], nucleic acid different from the native CCMV RNA [16] and other negatively charged particles [17]. In all cases, these VLPs formation are leaded by electrostatic interactions mediated by $\mathrm{pH}$ and $\mathrm{I}$.

On the other hand, gold nanoparticles have been studied due to their unique optical and electronic properties, and have been the subject of substantial research, with applications in a wide variety of areas, including electronics, nanotechnology, and biomedicine [18]. The properties of these nanoparticles, and therefore their applications, depend strongly on their size and shape [19]. Different shapes of gold nanoparticles have been synthetized such as nanospheres [20], nanorods [21], nanostars [22], nanotubes [23], nanocubes [24], nanodisks, nanowires [25], and nanoshells [26]. One of the main features of these nanoparticles is that their absorption peak maximum depends on their morphology, which is not only of scientific but also of technological interest. One of the most interesting applications is in the so-called area of nanomedicine. For example, gold nanorods and nanoshells have their absorption peak located in the transparent window of biological tissues; this is, in the near infrared region (NIR), from about 750 to $1200 \mathrm{~nm}$ [27]. For such particles, their strong surface plasmon absorption can convert NIR radiation into heat [28], which is favorable for their use in photothermal therapies for cancer treatment [29], photothermally triggered drug release [30], and gene therapy [31]. In addition, their strong scattering also makes them good contrast agents for medical imaging [32].

Various groups have encapsidated solid spherical gold nanoparticles with viral capsids [33] encapsidated solid AuNP, with sizes ranging from 5 to $15 \mathrm{~nm}$, with the red clover necrotic mosaic capsid protein. They used a strategy consisting in tethering the oligomers of viral DNA-2 to the Au nanoparticles, at conditions that limit the oligomers bound per particle, so the RNA-1 binds to this oligomeric DNA to induce the self-assembly of the capsid protein, through genome-CP interactions, capturing in the process the gold nanoparticle into the capsid. Dragnea's group has also conducted extensive research on packaging solid spherical AuNP of different sizes. They reported the selfassembly of viral protein around a core of gold in the absence of its nucleic acid [34]; they encapsidate citrate coated gold nanoparticles of about 2.5-4.5 nm in diameter, adding brome mosaic virus (BMV) purified capsid protein, which form a capsid around the particles where the interaction forces that create these VLPs are purely electrostatic [1]. They also use $12 \mathrm{~nm}$ AuNP functionalized with hydrophilic triethylene glycol (TEG). After functionalization of the particles, the diameter of the construct increased to about $16 \mathrm{~nm}$, which approximates the inner diameter of the viral capsid of the BMV virus. The size of the VLPs obtained was $26 \pm 2 \mathrm{~nm}$ in diameter which is identical in size to the native virus. They also conducted a study on the self-assembly of BMV capsid proteins around spherical gold nanoparticles (AuNP) functionalized with polyethylene glycol (PEG) [35], which is also a hydrophilic polymer. They successfully form VLPs with these AuNP, they use a ratio of 1:270 AuNP:proteins and AuNP diameters of 6, 9, 12, 15 and $18 \mathrm{~nm}$ after functionalization with PEG. They proposed that nanoparticles functionalized with PEG can be used as efficient nucleation centers for the formation of VLPs. They also report that the self-assembly efficiency is also a function of the gold core diameter, and peaks with the functionalized cores of $16 \mathrm{~nm}$, which is about the size of the interior diameter of the native virus. They also report the encapsidation of TEG functionalized AuNP with CCMV using gold cores of 6 and $12 \mathrm{~nm}$ in diameter coated with thiolalkylated tetra(ethylene glycol) (TTEG). They, however, used a mutant of CCMV CP lacking most of the N-terminal domain. They conclude that is possible to encapsidate AuNP with a cleaved protein lacking its amino terminal, which results in an increased flexibility in the dihedral angle of the dimer, resulting in capsids that can vary in size and structure capable of encapsidating different core size [36]. 
In order to increase gold nanoparticles biocompatibility, monodispersity and enhancing the surface in which a ligand can be added to provide specificity to the nanoparticle, we propose the encapsidation of gold nanoparticles with the purified native capsid protein of CCMV virus. Therefore, we present a procedure for the encapsidation of different types of gold nanoparticles; solid spherical gold nanoparticles, ultrasmall gold nanoshells and gold nanorods. Although functionalized gold nanoparticles with Bis-p-(sufonatophenyl)phenyl Phosphine (BSPP) ligands have been encapsidated with CCMV [37]. We show for the first time the direct use of wild type CCMV capsid proteins, not only for the encapsidation of spherical-shaped gold nanoparticles but also with rod-like shapes without ligands. Gold nanoshells and gold nanorods have advantages and disadvantages for medical applications; both can be used for phototermal therapy and imaging contrast. However, gold nanoshells are highly unstable and they last for about two-weeks before they break-up due to an Ostwald ripening process [38]. While the synthesis of gold nanorods produce them with a highly positive surface charge, which is highly cytotoxic [39]. These gold nanoparticles have shown great potential in medical applications and their encapsidation can improve their stability, increase their lifetime, and enhance biocompatibility, as well as producing monodisperse VLPs, and create a new substrate around the nanoparticle which can be used for ligand attachment to produce specific VLPs [40] with desirable qualities.

\section{Results}

Typical images of the different synthetized gold nanoparticles were observed with scanning electron microscopy (TEM). The morphology of both the $5 \mathrm{~nm}$ and $\mathrm{nm} 20 \mathrm{~nm}$ diameter nanospheres, with nanoparticles having a quasi-spherical shape, are shown in Figs. 2a) and 2b). Gold nanorods are shown in Fig. 2c), where we can observe that they also have a regular morphology. Figure 2d) shows an image of the gold nanoshells.

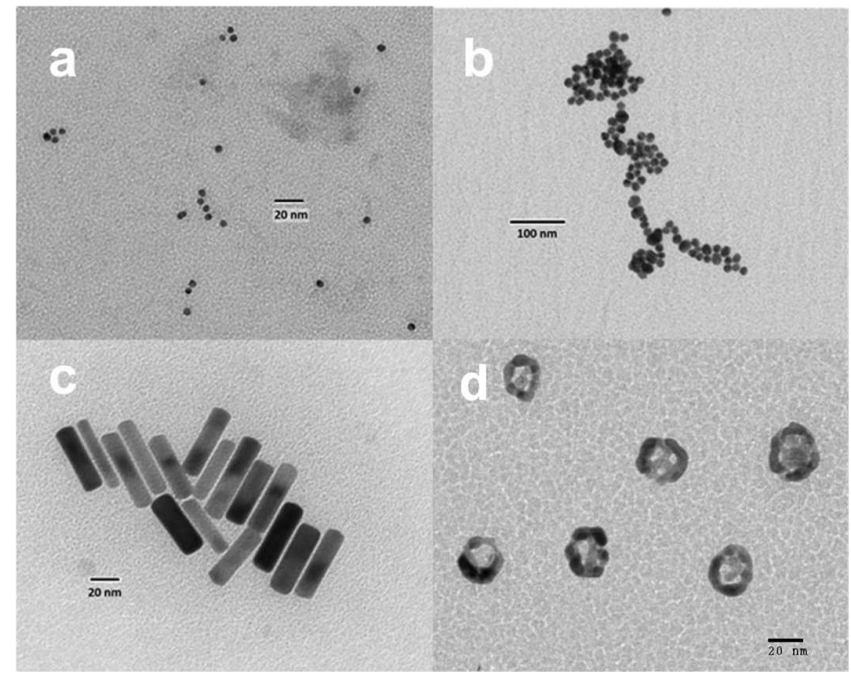

Figure 2. Images of AuNP a). $5 \mathrm{~nm}$ and b) $18 \mathrm{~nm}, \mathrm{c})$. AuNRs and d). AuNSs.

Figure 3 shows the absorption spectra of the Au NP (18 nm), AuNR and AuNSs. The AuNPs exhibit the characteristic absorption peak for their size in the visible region, at about $525 \mathrm{~nm}$. Both the AuNRs and the AuNSs show at absorption en the NIR region: The AuNRs shows a broad peak with a maximum at about $780 \mathrm{~nm}$, while the AuNSs shows a more defined peak with a maximum at about $815 \mathrm{~nm}$. 


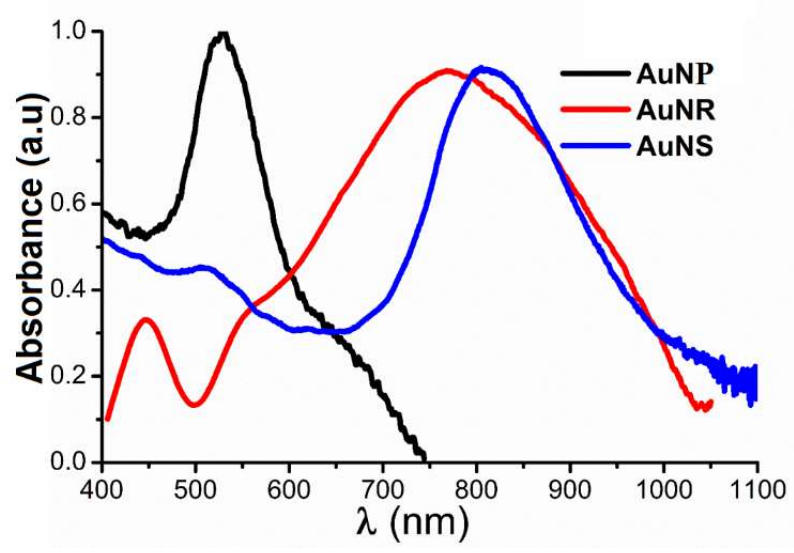

Figure 3. Typical UV-Vis-NIR absorption spectra of AuNPs (18 nm), of AuNR and AuNS. Note that the spectra of the AuNR and AuNS peak at the near infrared region, around $800 \mathrm{~nm}$.

AuNPs of $18 \pm 0.3 \mathrm{~nm}$ diameter with a surface charge of $-49 \mathrm{mV}$ (obtained by dynamic light scattering, not shown) were successfully encapsidated by the CCMV CP as it can be seen in Figure 4. All the AuNPs have a capsid, at $\mathrm{pH}=4$ and $\mathrm{I}=0.1$, and with the correct molarity of protein; this was expected since the particles have a highly negatively surface charge, which interact with the positively charged N-terminus of the protein leading to a $100 \%$ encapsidation. At this conditions empty capsids also form, because of the excess of CCMV CP we can observe several empty capsids. At $\mathrm{pH}=7$ and $\mathrm{I}=0.1$ the encapsidation is also highly efficient because the negative charge of the AuNPs is also quite high, but no empty capsid can be seen, since at this conditions tubes are the equilibrium structures, however, a very high concentration of CCMV CP is required to form empty tubes [8]. In this case, we conclude that the spherical shape of the nanoparticles forces the $\mathrm{CP}$ to self-assemble into spherical capsids around the nanoparticles.
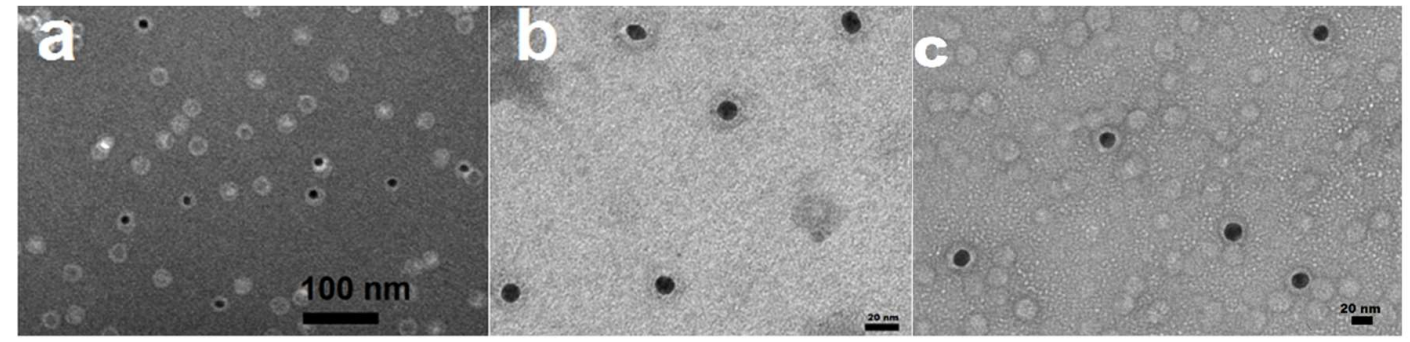

Figure 4. $18 \mathrm{~nm}$ AuNP encapsidated by the CCMV CP at different conditions a) $\mathrm{pH}=3.6$ and $\mathrm{I}=0.1 \mathrm{M}$. b) $\mathrm{pH}=7$ and $\mathrm{I}=0.1 \mathrm{M}$ and $\mathrm{c}) \mathrm{pH}=4$ and $\mathrm{I}=0.02 \mathrm{M}$.

On the other hand, several $5 \mathrm{~nm}$ AuNPs can be encapsidated in a single capsid, as shown in Fig. 5, at $\mathrm{pH}=4$ and $\mathrm{I}=0.1 \mathrm{M}$. It can be observed in Fig. 5a that the capsids can contain from one up to about 10 nanoparticles. Even more, a high number of nanoparticles can be trapped in a multicapsid structure, as shown in Fig. 3d, similar to those found in the formation of VLPs with very long RNA cores [16]. 


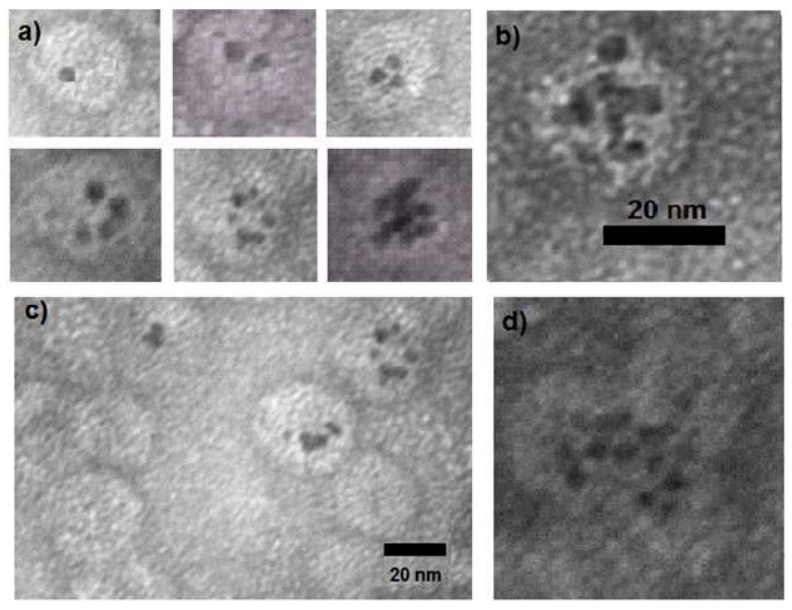

Figure $5.5 \mathrm{~nm}$ AuNPs encapsidated by the CCMV CP at $\mathrm{pH}=4$ and $\mathrm{I}=0.1 \mathrm{M}$. The black dots are the AuNPs.

The synthetized AuNS have an average diameter of $25 \pm 5 \mathrm{~nm}$ and an average surface charge average of $-22 \mathrm{mV}$ at $\mathrm{pH}=4$. At this $\mathrm{pH}$ the CCMV CP total charge is slightly negative [8], but still the arginine tails are expected to interact with the AuNSs; therefore, at these conditions, it was expected that the CCMV CP and the AuNSs will interact electrostatically. Note that the selected conditions for the encapsidation are in the limit of single capsid and multicapsid formation. We chose this condition due to many AuNSs have a diameter greater than the inner diameter of a single capsid. In this way, we want to secure that the AuNS will be completely covered by the CCMV CP. As we can see in Figure 6, all the AuNS were encapsidated, although due to the experimental conditions of the encapsidation, and due to the excess of capsid protein, we can also observed the formation of empty capsids of different sizes (see Fig. 6c).
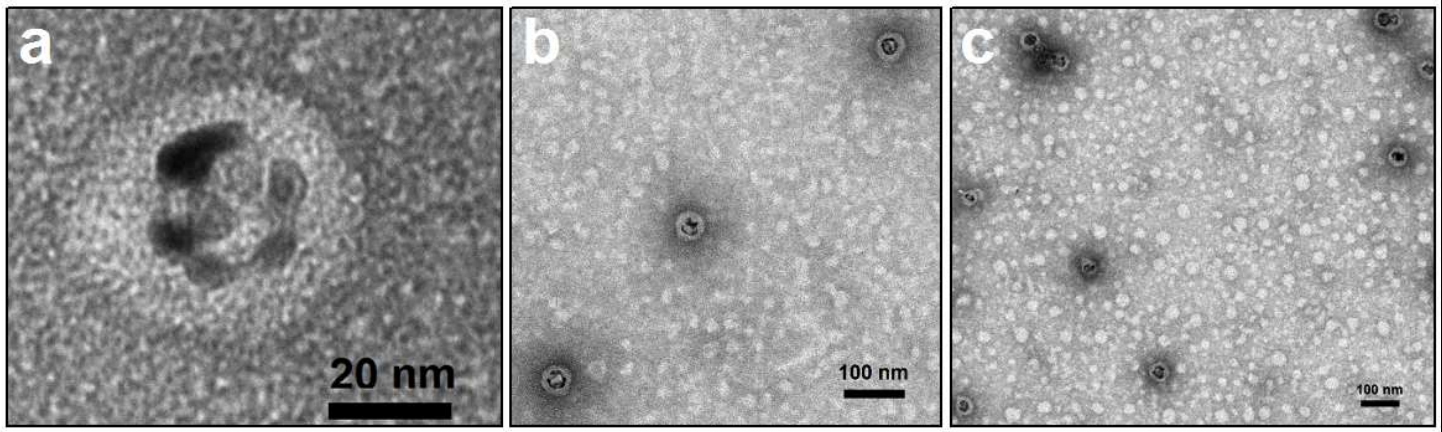

Figure 6. a). and b). AuNSs encapsidated with CCMV CP. c). A mixture of VLPs with AuNSs cores with CCMV CP and empty capsids of CCMV CP.

As mentioned above, the diameter of the AuNSs exceed the $21 \mathrm{~nm}$ inner diameter of the native virus, because of that the capsids formed around most of the AuNS are multishell capsids, creating VLP with average diameter of $43.9 \pm 6.4 \mathrm{~nm}$. The CCMV CP is forced to self-assemble into spherical shells that are not perfect icosahedra $[8,11]$. Consequently, the distribution of protein conformations on larger particles is significantly different than in the wild-type capsid. This has also been observed in the encapsidation of large nanoemulsion droplets with the CCMV CP [41].

The AuNRs synthetized for this work have an average size of $50.8 \times 12.5 \mathrm{~nm}$, with an aspect ratio of $4.1 \pm 0.6 \mathrm{~nm}$ and a maximum absorbance peak at $\sim 780 \mathrm{~nm}$ (see Fig. 3). After the synthesis process, the AuNRs have an average zeta potential of $20 \mathrm{mV}$ determined by electrophoresis measurements using dynamic light scattering. The treatment with PA changed the surface charge of the AuNRs from positive to negative, to an average zeta potential value of $-63.8 \mathrm{mV}$. Figures $7 \mathrm{~b}$ and $7 \mathrm{c}$ show TEM images of the AuNRs before and after treatmet with PA. 

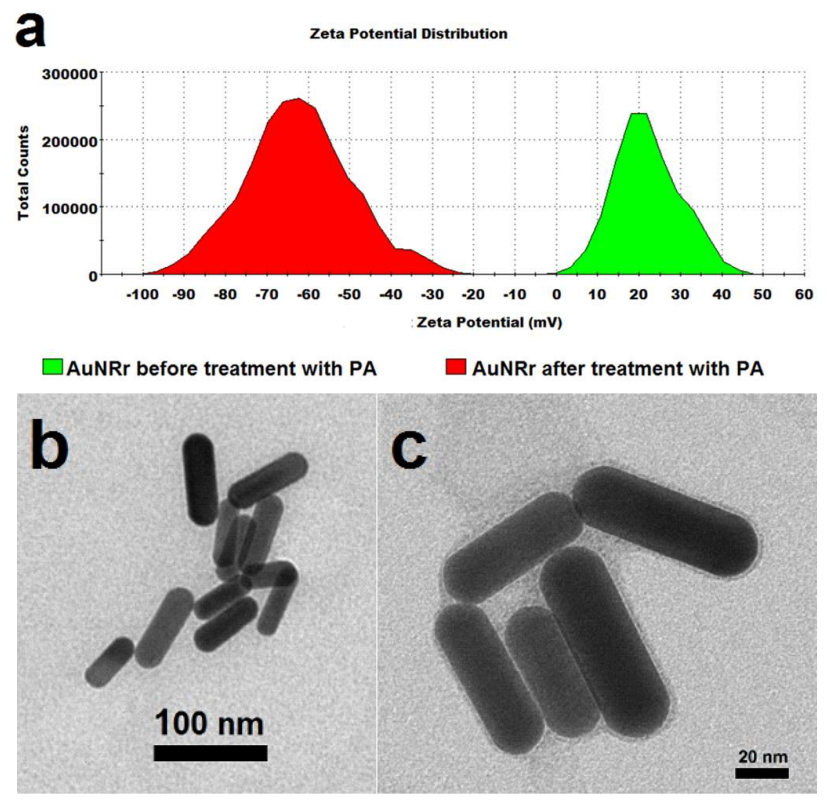

Figure 7. a. Zeta potential of AuNRs before and after treatment with PA. b. AuNRs after synthesis. c. AuNRs after treatment with PA.

After mixing the AuNRs with the CCMV CP, we found that the AuNRs are encapsidated in two ways; one where the smaller nanorods are enclosed in a single nonsymmetrical capsid, while in the longer nanorods the proteins form a nanotube capsid along the longer axis and in the tips form icosahedral structures as it is shown in Figure 8a-c.
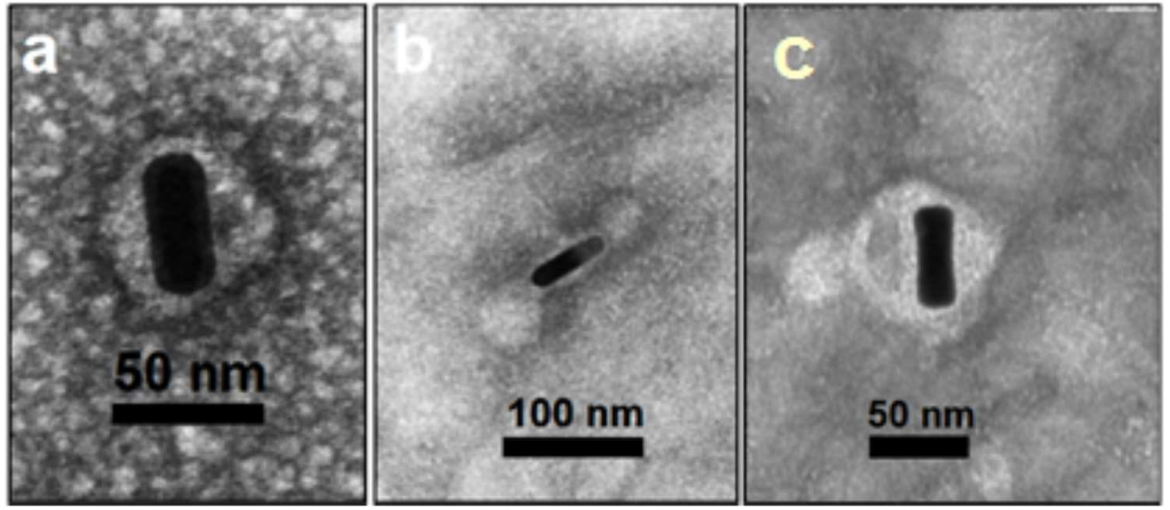

Figure 8. Gold Nanorods encapsidated by the capsid protein of CCMV.

\section{Discussion}

Here, we show the formation of VLPs that contain plasmonic gold nanoparticles of different shapes, such as gold nanospheres, nanorods and nanoshells. These nanoparticles have physical properties that are of interest in nanomedicine, as imaging contrast agents and photothermal therapy.

Since the quasi-spherical AuNPs have a highly negatively surface charge, they interact with the positively charged $\mathrm{N}$-terminus of the protein leading to a $100 \%$ encapsidation. At low $\mathrm{pH}$ empty capsids also form due to at this conditions almost all the protein put into the system self-assembly into capsids; because of the excess of CCMV CP we can observe several empty capsids. However, at $\mathrm{pH}=7$ and $\mathrm{I}=0.1$ the encapsidation is also highly efficient. This was surprising because at this 
condition the formation of protein nanotubes is the preferred self-assembly structure. In addition, at these condition a high concentration of protein is needed to form any self-assembly structure. In this case, we conclude that the spherical shape and the high negative charge of the nanoparticles forces the $\mathrm{CP}$ to self-assemble into spherical capsids around them. In addition, at these conditions no empty capsid can be observed, since at this conditions protein nanotubes are the equilibrium structures, but a very high concentration of CCMV CP is required to form empty tubes [8]. In the case of the small quasi-spherical AuNPs, we found that a different number of particles can be encapsidated. Even more, sometimes we observed the formation of jointed multicapsids that contain a large number of these small nanoparticles.

The AuNSs are very interesting nanoparticles since they absorb in the NIR region, and they have a great potential for using them in photothermal therapy. The experimental conditions, at which the AuNSs were encapsidated, were selected to try to prevent partial encapsidation, especially for the larger capsids. However, we found that all AuNSs were encapsidated, surprisingly, by a single protein shell; this conclusion was determined from the thickness of the protein layer around the AuNSs, which is $\sim 7 \mathrm{~nm}$. In the case of the AuNSs, even though their average diameter might be greater that the inner diameter of the native virus, the electrostatic interaction between the CCMV $\mathrm{CP}$ with the gold nanoparticles is strong enough to follow the shape and size of the nanoparticle. However, it is known that the CCMV capsid is quite flexible and it is capable of encapsidating larger genomes than its natural genome [16], and the larger AuNSs might be a similar case. A draw back on the use of AuNSs is its well-known instability because in short periods of time they become fragmented due to an Ostwald ripening process [38]; Ostwald ripening leads to the dissolution of smaller nanoparticles into larger ones through coalescence to decrease the interfacial energy between the nanoparticles and the solvent. Colloid nanoparticles become increasingly unstable with decreasing particle size due to the relative increase in higher energy surface area. The solubility concentration of the particle is directly proportional to the surface tension and inversely proportional to the radius of the nanoparticle. Therefore, the AuNSs are destroyed by transferring of gold atoms from smaller shells to larger ones. Furthermore, gold nanoshells are susceptible to photothermal fragmentation, when exposed to high IR incident intensities [42]. It has been shown that encapsidation by the CCMV capsid can provide stability against Ostwald ripening in nanolipospheres systems [43]. Therefore, the encapsidation can provide not only a biocompatible surface but also an envelope that can give them a better stability against fragmentation either due to Ostwald ripening or photofragmentation.

On the other hand, AuNRs are the better known nanoparticles for photothermal therapy applications. As the other gold nanoparticles in solution, these nanorods need to be highly charged to avoid aggregation. One of the most known synthesis method produce them with a high positive surface charge. It has been shown that particles with a high positive charge are undesirable because they are cytotoxic. Therefore, we developed a method to change the surface charge of the AuNRs from positive to negative charge, to make them more biocompatible. Even though the encapsidation of the AuNRs was also successful, the shape of the capsids around them was not uniform, as in the case of the AuNPs and AuNSs.

In general, we show that it was possible to encapsidate these types of gold nanoparticles with the capsid protein of the plant virus CCMV, without surface modification of the nanoparticles as has been reported $[35,36]$. We vary the experimental conditions; this is, we varied $\mathrm{pH}$, ionic strength and concentration of the CCMV CP conditions. We found that the encapsidation efficiency was $100 \%$ in all cases.

\section{Materials and Methods}

Silver nitrate $\left(\mathrm{AgNO}_{3} ; 99.99 \%\right)$, sodium borohydride $\left(\mathrm{NaBH}_{4} ; 99 \%\right)$, trisodium citrate dihydrated ( $\mathrm{NaCit} ; 99 \%)$, potassium carbonate (K2CO3; 99\%), cloroauric acid $\left(\mathrm{HAuCl}_{4} ; 99.9 \%\right)$, hexadecyltrimethylammonium bromide $\left(\mathrm{C}_{19} \mathrm{H}_{42} \mathrm{BrN} ; 99 \%\right)$, hydrogen peroxide $(\mathrm{H} 2 \mathrm{O} 2 ; 30 \%)$ Phenylmethylsulfonylfluoride (PMSF; 99\%) calcium chloride $\left(\mathrm{CaCl}_{2} ; 99 \%\right)$, citric acid $(\mathrm{C} 6 \mathrm{H} 8 \mathrm{O} 7$; 
99.5\%), dithiothreitol (DTT;99\%), Tris(hydroxymethyl)aminomethane (Tris; 99.8\%) (Sigma Aldrich, USA), sodium hydroxide ( $\mathrm{NaOH} ; 97 \%$ ) (Fisher BioReagents, USA), sodium chloride (NaCl; $100 \%)$ (JT Baker, USA), ethylenediaminetetraacetic acid (EDTA; 99\%, Bio Rad, USA),L- $\alpha$-phosphatidic acid (PA; 99\%) (Avanti Polar Lipids, USA). All materials were used without further purification. Stock solutions of sodium borohydride, sodium citrate, and silver nitrate were freshly prepared for each reaction. Deionized bioresearch grade, and sterilized milliQ-type water was used for all reactions.

4.1 Gold nanoparticles synthesis.

AuNP. The spherical gold nanoparticles were synthetized by the inverted Turkevich method [44], which consist in a reduction of a gold salt $\mathrm{HAuCl} 4$ with sodium citrate as a reduction agent at its boiling temperature. In order to obtain the desired size of $18 \mathrm{~nm}$, we use a molar ratio of $3.4 \mathrm{M}$ $\mathrm{HAuCl} 4$ :sodium citrate, and for the $5 \mathrm{~nm}$ particles we use a molar ratio of $20 \mathrm{M} \mathrm{HAuCl4:sodium}$ citrate; this procedure produce highly monodisperse particles.

AuNSs. For the synthesis of AuNSs, we use a combination of the seed-mediated growth using the Lee-Meisel methods, which consists of a thermal reduction with citrate [45].

Seed solution. $2 \mathrm{~mL}$ of $1 \% \mathrm{NaCit}$ was mixed with $7.6 \mathrm{~mL}$ of water and vigorously stirred while heating at $70{ }^{\circ} \mathrm{C}$ for $15 \mathrm{~min}$. Then, $170 \mu \mathrm{L}$ of AgNO3 $1 \%$ solution and $200 \mu \mathrm{L}$ at $4{ }^{\circ} \mathrm{C}$ of $\mathrm{NaBH} 40.1 \%$ solution were added at the same time, while stirring and heating were continued for $60 \mathrm{~min}$, ultimately obtaining $4 \mathrm{~nm}$ nanoparticles in diameter.

Growth of AgNPs. In a round bottom flask, $400 \mu \mathrm{L}$ of NaCit $1 \%$ solution and $16 \mathrm{~mL}$ of water were mixed with vigorous stirring and was brought to its boiling temperature for $15 \mathrm{~min}$. Then $2 \mathrm{~mL}$ of the seed solution and $340 \mu \mathrm{L}$ of AgNO3 1\% solution were added while stirring and heating continued for 60 more min; the resulting solution was kept at room temperature in darkness.

AuNSs preparation. The gold layer was formed onto the AgNP by deposition in a procedure adapted from Vongsavat et al. [46], and improved by Duran-Meza et al. [45] that involves the preparation of a potassium-gold (K-gold) solution and then mixing the AgNPs with the K-gold solution.

K-gold Solution and Gold deposition. In a round bottom flask covered from light, $10 \mathrm{~mL}$ of deionized water and $2.5 \mathrm{mg}$ of $\mathrm{K} 2 \mathrm{CO} 3$ were added, then $200 \mu \mathrm{L}$ of $1 \%$ solution of $\mathrm{HAuCl} 4$ was incorporated to the mixture under vigorous stirring at room temperature for $30 \mathrm{~min}$. The resulting solution was kept for 12 hours at $4{ }^{\circ} \mathrm{C}$ before use. However, we discovered that the thickness of the gold shell can be modified by varying the reaction time and/or the volume ratio of the K-gold to the AgNP solutions [39]: The K-gold solution was added into the AgNPs solution at a volume ratio of 1:1 to obtain a $25 \pm 5 \mathrm{~nm}$ AuNSs with a $4 \mathrm{~nm}$ thickness that have a maximum absorption peak in the near infrared at $805 \mathrm{~nm}$. In a $5 \mathrm{~mL}$ vial the AgNP were mixed with the K-gold solution, then it was stirred for 5 minutes at room temperature. To clean the AuNSs, the resulting solution was centrifuged at $6000 \mathrm{~g}$ (Biofuge Stratus, Heraus, Germany) for 10 minutes, and the sediment was resuspended in water. The centrifugation and resuspension process was repeated three times and the solution was kept at room temperature.

AuNRs. We prepare AuNRs by the method developed by Xu et al. [47] that produces a wellcontrolled morphology, high purity, and good quality monodisperse rods. This method involves the preparation of a gold seed solution and the growing of the AuNRs at alkaline conditions.

Seed preparation. HAuCl4 was added to a CTAB solution, and then $\mathrm{NaBH} 4$ was added to the solution while stirring. The final concentration of the seed solution was 5x10-3 M HAuCl4, $0.2 \mathrm{M}$ $\mathrm{CTAB}$ and $0.01 \mathrm{M} \mathrm{NaBH}$. The stirring was stopped after $2 \mathrm{~min}$, and then the solution was aged for at least 2 hours before use at room temperature.

AuNRs Growth and its Functionalization. We used $10 \mathrm{~mL}$ of a solution composed of $5 \times 10-3 \mathrm{M}$ HAuCl4, 0.1 M CTAB, 5x10-3 M NaOH, 0.02 M H2O2, $0.06 \mathrm{nM}$ AgNO3 and $15 \mu \mathrm{L}$ of the seed solution. The resulting solution was centrifuged at $6000 \mathrm{~g}$ for 10 minutes and resuspended in water. The process was repeated and the solution was kept at room temperature. AuNRs prepared by this procedure usually presents an absorbance peak in the NIR region, which can be compared in their thermal absorbance with the AuNSs [45]. AuNRs synthetized by this method produce highly positively charged AuNRs due to the stabilizing surfactant bilayer made out of CTAB. However, a highly positively charged nanorods surface is undesirable because it is cytotoxic [39]. In addition, it 
will not interact appropriately with the CCMV CP; therefore, the AuNRs require a change of the superficial charge from positive to negative, so the positively charged $\mathrm{N}$-terminus of the CCMV CP can interact with the AuNRs and self-assemble around them. In order to make this surface charge change, we use a volume ratio 1:1 of $0.02 \mathrm{M}$ AuNRs solution and $1 \mathrm{M}$ PA while sonicated for $10 \mathrm{~min}$. The above solution was stored at $4{ }^{\circ} \mathrm{C}$ for $24 \mathrm{hrs}$. The PA is a biocompatible anionic lipid, which forms a lipid bilayer on the AuNPs, replacing the $\mathrm{CTAB}$ on the nanorods surface; since CTAB is not covalently attached to the nanorods surface, reversing in this way their surface charge. The final superficial change, from positive to negative, was monitored by micro electrophoresis with Dynamic Light Scattering (DLS) in a Zetasizer Nano ZS (Malvern Instruments, England).

CCMV capsid protein purification. CCMV was harvested and purified from infected Cowpea plants (Vigna ungiculata) following established protocols [4,48]. Briefly, it was dialyzed against disassembly buffer (500 mM CaCl2, $50 \mathrm{mM}$ Tris- $\mathrm{HCl} \mathrm{pH}=7.5,1 \mathrm{mM}$ EDTA, $1 \mathrm{mM}$ DTT, and $0.5 \mathrm{mM}$ PMSF) for 24 hours in order to disrupt the viral capsids and separate the capsid protein and the RNA. Then the disassembled virus was ultracentrifuged, at 100,000 rpm using a TLA110 rotor for $106 \mathrm{~min}$ at $4{ }^{\circ} \mathrm{C}$, in order to precipitate the RNA and recover the purified protein still in suspension. The recovered protein was then dialyzed in a protein disassembly buffer $(1 \mathrm{M} \mathrm{NaCl}, 50 \mathrm{mM}$ Tris- $\mathrm{HCl} \mathrm{pH}$ = 7.2, 1 mM EDTA, $1 \mathrm{mM}$ DTT, $1 \mathrm{Mm}$ PMSF) [49]. Protein concentration and purity was measured in a UV-Vis spectrophotometer (NanoDrop 2000c UV-Vis Spectrophotometer, Thermo Fisher Scientific Inc., Waltham, MA, USA), and only the fractions with 280/260 ratios above 1.5 were chosen and used for the encapsidation experiments.

AuNPs encapsidation. Capsid formation around the nanoparticles was performed during $24 \mathrm{hr}$ microdialysis for each of the gold nanoparticles used at each particular condition shown in Fig. 1. AuNP of $18 \mathrm{~nm}$ were encapsidated in three different conditions, citrate buffer (citric acid/sodium citrate) $\mathrm{pH}=4, \mathrm{I}=0.02 \mathrm{M}$, citrate buffer $\mathrm{pH}=3.6, \mathrm{I}=0.1 \mathrm{M}$ and phosphate buffer (sodium phosphate monobasic/sodium phosphate dibasic) $\mathrm{pH}=7, \mathrm{I}=0.1 \mathrm{M}$. In all cases, the ratio of 540 protein per particle was used, favoring the formation of icosahedral capsids. AuNP of $5 \mathrm{~nm}$ were encapsidated in citrate buffer $\mathrm{pH}=4$ and $\mathrm{I}=0.1 \mathrm{M}$, and at a ratio of 540 protein per 5 particles [11]. AuNSs were encapsidated in citrate buffer at $\mathrm{pH}=4$ and $\mathrm{I}=0.01 \mathrm{M}$, and also a ratio of 540 proteins per particle was used. In order to promote the formation of protein tubes around the AuNRs, a phosphate buffer at $\mathrm{pH}=6.5, \mathrm{I}=0.01 \mathrm{M}$ and a ratio of 960 proteins per particle was used.

Characterization methods. The size and morphology of the AuNP, AuNSs, and AuNRs were characterized by transmission electron microscopy (TEM), using a JEOL 1230 instrument, at $100 \mathrm{kV}$. The samples were prepared by depositing an $8 \mu \mathrm{L}$ drop onto a copper grid (400 mesh) that was previously coated with parlodion and carbon. After $1 \mathrm{~min}$, the excess sample was removed with a filter paper and then it was negatively stained with uranyl acetate $2 \%$ for 60 seconds and dried off again; the grid was stored in a desiccator overnight. The images were processed using the ImageJ program [50], the size distribution histograms were constructed from the analysis of at least 200 particles, each nanoparticle was measured in two directions. The absorption extinction spectra curves were measured by UV-Vis and IR Spectroscopy at room temperature on a Spectrophotometer UVVis (Nanodrop 2000, Thermo Scientific, USA). Zeta potential was measured by electrophoresis in a Zetasizer (Nano ZS, Malvern Instrument, England).

\section{Conclusions}

We report the formation of VLPs that contain plasmonic gold nanoparticles of different shapes and physical properties that are of interest in nanomedicine. Among them are gold nanospheres, nanorods and nanoshells. It is important to mention that it was possible to encapsidate these types of gold nanoparticles with the complete capsid protein of CCMV and without the need of capping the particles with TEG or PEG [35,36], under different $\mathrm{pH}$, ionic strength and concentration of the CCMV $\mathrm{CP}$ conditions. As it was expected, at $\mathrm{pH}=3.6$ we can see the formation of empty capsids as well, since at this $\mathrm{pH}$ almost all the capsid protein is expect to go onto the formation of capsids. At $\mathrm{pH}=7$, we did not observed the formation of empty capsids because at this condition the concentration of the CCMV CP required for the formation of empty capsid is extremely high [8]; however, the presence 
of a negatively charged core, such as the AuNPs, the CP interaction with the core is strong enough to drive the self-assembly of the CP around the core to form an spherical VLP at this condition. This result is surprising for two reasons; first, at this high $\mathrm{pH}$ and $\mathrm{I}$, the concentration of protein require for self-assembling into empty structures is much higher, and second, at this conditions the preferred self-assembled structure of the CP is tubular. Therefore, our results show that the interaction of the CCMV CP with a negative core is strong enough to drive it's self-assembly into the shape of the core.

In the case of the AuNSs, even though their average diameter might be greater that the inner diameter of the native virus, the electrostatic interaction between the CCMV CP with the gold nanoparticles is strong enough to follow the shape and size of the nanoparticle. However, it is known that the CCMV capsid is quite flexible and it is capable of encapsidating larger genomes that it's natural genome [16]. In addition, encapsidating them in the conditions were multishells wall capsids are formed, secures that the AuNSs is completely surrounded by protein, which lead to an encapsidation efficiency of $100 \%$ of the AuNSs: The VLP formed with this procedure have a mean diameter of $43.9 \mathrm{~nm}$. Furthermore, the encapsidation might help to stabilize the AuNSs against fragmentation due to either Ostwald ripening or photofragmentation [38,42]. Because of the excess of the CCMV CP empty capsids are also formed. It is important to emphasize that these nanoparticles are nontoxic for use in Vivo therapies [51]. The VLP formed with the AuNRs are encapsidated in two different manners, in a capsid with an oval shape and in tubes with icosahedral capsids at the tips of the tubes. The shape of the encapsidating structures depends on the length of the nanorods. The AuNRs require a change of charge, because of the synthesis method produce them with high positive surface charge, which is cytotoxic. Here, we show a procedure to reverse the surface charge of the nanorods from positive to negative charge that makes them more biocompatible.

In summary, we show that it was possible to encapsidate these types of gold nanoparticles with the capsid protein of the plant virus CCMV, without surface modification of the nanoparticles. We vary the experimental conditions; this is, we varied $\mathrm{pH}$, ionic strength and concentration of the CCMV CP conditions, and found $100 \%$ encapsidation efficiency in all cases. Even more, the capsid protein can be easily functionalized to give them specific cell targeting ability.

\section{Author Contributions:}

A. L. Duran-Meza carried out the experiments, wrote and discussed the paper; M. I. Escamilla-Ruiz helped with the synthesis, characterization and encapsidation with CCMV CP of gold nanorods; X. F. Segovia-González helped with the synthesis, characterization and encapsidation with CCMV CP of gold nanoshells; M. V. Villagrana-Escareño helped with the purification and characterization of CCMV and CCMV CP; J. R. VegaAcosta directed the experiments and J. Ruiz-Garcia directed the project, wrote and discussed the paper and funding acquisition.

Funding: This research was funded by CONACYT, grant numbers PN-248787, CB-254981, CB-237439, and FC341. Ana Luisa Duran-Meza also acknowledges support from UC-MEXUS-CONACYT.

Acknowledgments: We thank Prof. Charles M. Knobler for helpful comments.

Conflicts of Interest: The authors declare no conflict of interest. The funders had no role in the design of the study; in the collection, analyses, or interpretation of data; in the writing of the manuscript, or in the decision to publish the results. 


\section{References}

1. Chen, C.; Daniel, M. C.; Quinkert, Z. T.; De, M.; Stein, B.; Bowman, V. D.; Chipman, P. R.; Rotello, V. M.; Kao, C. C.; Dragnea, B. Nanoparticle-templated assembly of viral protein cages. Nano Lett. , 2006, 6, 611-615. DOI: 10.1021/nl0600878.

2. Bancroft, J. B.; Bracker, C. E.; Wagner, G. W. Structures derived from cowpea chlorotic mottle and brome mosaic virus protein. Virology 1969, 38, 324-335. DOI: 10.1016/0042-6822(69)90374-2.

3. de la Escosura, A.; Nolte, R. J. M.; Cornelissen, J. J. L. M. Viruses and protein cages as nanocontainers and nanoreactors. J. Mater. Chem. 2009, 19, 2274-2009. DOI: 10.1039/B815274H.

4. Wyatt, S. D.; Kuhn, C. W. Replication and properties of cowpea chlorotic mottle virus in resistant cowpeas. Phytopathology 1979, 69, 125-129. DOI: 10.1094/Phyto-69-125.

5. Bancroft, J. B.; Hiebert, E.; Rees, M. W.; Markham, R. Properties of cowpea chlorotic mottle virus, its protein and nucleic acid. Virology 1968, 34, 224-239. DOI: 10.1016/0042-6822(68)90232-8.

6. Bancroft, J. B.; Hills, G. J.; Markham, R. A study of the self-assembly process in a small spherical virus. Formation of organized structures from protein subunits in vitro. Virology 1967, 31, 354-379. DOI: 10.1016/0042-6822(67)90180-8.

7. Zlotnick, A.; Mukhopadhyay, S. Virus assembly, allostery and antivirals. Trends Microbiol. 2011, 19, 14-23. DOI: 10.1016/j.tim.2010.11.003.

8. Vega-Acosta, J. R.; Cadena-Nava, R. D.; Gelbart, W. M.; Knobler, C. M.; Ruiz-Garcia, J. Electrophoretic Mobilities of a Viral Capsid, Its Capsid Protein, and Their Relation to Viral Assembly. J. Phys. Chem. B 2014, 118, 1984-1989. DOI: 10.1021/jp407379t.

9. Kaiser, C.R.; Flenniken, M. L.; Gillitzer, E.; Harmsen, A.L.; Harmsen, A. G.; Jutila, M. A.; Young, M. J. Biodistribution studies of protein cage nanoparticles demonstrate broad tissue distribution and rapid clearance in vivo 2007. International Journal of nanomedicine, 2(4), 715-733.

10. Adolph, K. W.; Butler, P. J. G.; Studies on the assembly of a spherical plant virus: I. States of aggregation of the isolated protein. J. Mol. Biol. 1974, 88, 327-341. DOI: 10.1016/0022-2836(74)90485-9

11. Lavelle, L.; Gingery, M.; Phillips, M.; Gelbart, W. M.; Knobler, C. M.; Cadena-Nava, R. D.; Vega-Acosta, J. R.; Pinedo-Torres, L. A.; Ruiz-Garcia, J. Phase diagram of self-assembled viral capsid protein polymorphs. J. Phys. Chem. B 2009, 113, 3813-3819. DOI: 10.1021/jp8079765.

12. G. Vriend.; B.J.M. Verduin. And M. A. Hemminga. Role of the N-terminal part of the coat protein in the assembly of cowpea chlorotic mottle virus: A $500 \mathrm{mhz}$ proton nuclear magnetic resonance study and structural calculations. Journal of Molecular Biology 1986. 191(3), 453-460.

13. Jolley, C.; Klem, M.; Harrington, R.; Parise, J.; Douglas, T. Structure and photoelectrochemistry of a virus capsid- $\mathrm{TiO}_{2}$ nanocomposite. Nanoscale 2011, 3, 1004-1007. DOI: 10.1039/c0nr00378f.

14. Hu, Y.; Zandi, R.; Anavitarte, A.; Knobler, C. M.; Gelbart, W. M. Packaging of a Polymer by a Viral Capsid: The Interplay between Polymer Length and Capsid Size. Biophys. J. 2008, 94, 1428-1436. DOI: 10.1529/biophysj.107.117473.

15. de la Escosura, A.; Verwegen, M.; Sikkema, F. D.; Comellas-Aragones, M.; Kirilyuk, A.; Rasing, T.; Nolte, R. J. M.; Cornelissen, J. J. L. M. Viral capsids as templates for the production of monodisperse Prussian blue nanoparticles. Chem. Commun. 2008, 1542-1544. DOI: 10.1039/B800936H.

16. Cadena-Nava, R. D.; Comas-Garcia, M.; Garmann, R. F.; Rao, A. L. N.; Knobler, C. M.; Gelbart, W. M. Self-Assembly of Viral Capsid Protein and RNA Molecules of Different Sizes: Requirement for a Specific High Protein/RNA Mass Ratio. J. Virol. 2012, 86, 3318-3326. DOI: 10.1128/JVI.06566-11.

17. Sánchez-Sánchez, L.; Cadena-Nava, R. D.; Palomares, L. A.; Ruiz-Garcia, J.; Koay, M. S.; Cornelissen, J. J.; Vazquez-Duhalt, R. Chemotherapy pro-drug activation by biocatalytic virus-like nanoparticles containing cytochrome P450. Enzyme Microb. Technol. 2014, 60, 24-31. DOI: 10.1016/j.enzmictec.2014.04.003.

18. Sardar, R.; Funston, A. M.; Mulvaney, P.; Murray, R. W. Gold Nanoparticles: Past, Present, and Future. Langmuir 2009, 25, 13840-13851. DOI: 10.1021/la9019475.

19. Daniel, M. C.; Astruc, D. Gold Nanoparticles: Assembly, Supramolecular Chemistry, Quantum-SizeRelated Properties, and Applications Toward Biology, Catalysis, and Nanotechnology. Chem. Rev. 2004, 104, 293-346. DOI: 10.1021/cr030698+.

20. Kimling, J.; Maier, M.; Okenve, B.; Kotaidis, V.; Ballot, H.; Plech, A. Turkevich Method for Gold Nanoparticle Synthesis Revisited. J. Phys. Chem. B 2006, 110, 15700-15707. DOI: 10.1021/jp061667w. 
21. Jana, N. R.; Gearheart, L.; Murphy, C. J. Wet Chemical Synthesis of High Aspect Ratio Cylindrical Gold Nanorods. J. Phys. Chem. B 2001, 105, 4065-4067. DOI: 10.1021/jp0107964.

22. Khoury, C.; Vo-Dinh, T. Gold nanostars for surface-enhanced Raman scattering: synthesis, characterization and optimization. J. Phys. Chem. C 2008, 112, 18849-18859. DOI: 10.1021/jp8054747.

23. Senger, R. T.; Dag, S.; Ciraci, S. Chiral single-wall gold nanotubes. Phys. Rev. Lett. 2004, 93, 1-5. DOI: 10.1103/PhysRevLett.93.196807.

24. Huang, C.-J.; Chiu, P.-H.; Wang, Y.-H.; Chen, W. R.; Meen, T. H. Synthesis of the Gold Nanocubes by Electrochemical Technique. J. Electrochem. Soc. 2006, 153, D129-D133. DOI: 10.1149/1.2203931.

25. Wang, B.; Yin, S.; Wang, G.; Buldum, A.; Zhao, J. Novel structures and properties of gold nanowires. Phys. Rev. Lett. 2001, 86, 2046-2049. DOI: 10.1103/PhysRevLett.86.2046.

26. Sun, Y.; Xia, Y. Mechanistic Study on the Replacement Reaction between Silver Nanostructures and Chloroauric Acid in Aqueous Medium Mechanistic Study on the Replacement Reaction between Silver Nanostructures and Chloroauric Acid in Aqueous J. Am. Chem. Soc. 2004, 126, 3892-3901. DOI: 10.1021/ja039734c.

27. Simpson, C. R.; Kohl, M.; Essenpreis, M.; Cope, M. Near-infrared optical properties of ex vivo human skin and subcutaneous tissues measured using the Monte Carlo inversion technique. Phys. Med. Biol. 1998, 43, 2465-2478. DOI: 10.1088/0031-9155/43/9/003.

28. Bashkatov, A. N.; Genina, E. A.; Kochubey, V. I.; Tuchin, V. V. Optical properties of human skin, subcutaneous and mucous tissues in the wavelength range from 400 to $2000 \mathrm{~nm}$. J. Phys. D. Appl. Phys. 2005, 38, 2543-2555. DOI: 10.1088/0022-3727/38/15/004.

29. Jain, S.; Hirst, D. G.; O'Sullivan, J. M. Gold nanoparticles as novel agents for cancer therapy. Br. J. Radiol. 2012, 85, 101-113. DOI: 10.1259/bjr/59448833.

30. Liang, Z.; Li, X.; Xie, Y.; Liu, S. 'Smart' gold nanoshells for combined cancer chemotherapy and hyperthermia. Biomed. Mater. 2014, 9, 025012. DOI: 10.1088/1748-6041/9/2/025012.

31. Huschka, R.; Barhoumi, A.; Liu, Q.; Roth, J. A.; Ji, L.; Halas, N. J. Gene silencing by gold nanoshellmediated delivery and laser-triggered release of antisense oligonucleotide and siRNA. ACS Nano 2012, 6, 7681-7691. DOI: 10.1021/nn301135w.

32. Huang, X.; El-Sayed, I. H.; Qian, W.; El-Sayed, M. A. Cancer Cell Imaging and Photothermal Therapy in the Near-Infrared Region by Using Gold Nanorods J. Am. Chem. Soc. 2006, 128, 2115-2120. DOI: 10.1021/ja057254a.

33. Loo, L.; Guenther, R. H.; Basnayake, V. R.; Lommel, S. A.; Franzen, S. Controlled encapsidation of gold nanoparticles by a viral protein shell. J. Am. Chem. Soc. 2006, 128, 4502-3. DOI: 10.1021/ja057332u.

34. Dragnea, B.; Chen, C.; Kwak, E.-S.; Stein, B.; Kao, C. C. Gold nanoparticles as spectroscopic enhancers for in vitro studies on single viruses. J. Am. Chem. Soc. 2003, 125, 6374-6375. Gold Nanoparticles as Spectroscopic Enhancers for in Vitro Studies on Single Viruses ... DOI: 10.1021/ja0343609.

35. Sun, J.; DuFort, C.; Daniel, M.-C.; Murali, A.; Chen, C.; Gopinath, K.; Stein, B.; De, M.; Rotello, V. M.; Holzenburg, A.; Kao, C. C.; Dragnea, B. Core-controlled polymorphism in virus-like particles. Proc. Natl. Acad. Sci. 2007, 104, 1354-1359. DOI: 10.1073/pnas.0610542104.

36. Aniagyei, S. E.; Kennedy, C. J.; Stein, B.; Willits, D. A.; Douglas, T.; Young, M. J.; De, M.; Rotello, V. M.; Srisathiyanarayanan, D.; Kao, C. C.; Dragnea, B. Synergistic effects of mutations and nanoparticle templating in the self-assembly of cowpea chlorotic mottle virus capsids. Nano Lett. 2009, 9, 393-398. DOI: $10.1021 / \mathrm{nl} 8032476$.

37. Liu, A.; Verwegen, M.; de Ruiter, M. V.; Maassen, S. J.; Traulsen, C. H. H.; Cornelissen, J. J. Protein cages as containers for gold nanoparticles. J. Phys. Chem. B 2016, 120, 6352-6357. DOI: 10.1021/acs.jpcb.6b03066.

38. Zhang, G. D.; Jasinski, J. B.; Howell, J. L.; Patel, D.; Stephens, D. P.; Gobin, A. M.; Tunability and stability of gold nanoparticles obtained from chloroauric acid and sodium thiosulfate reaction. Nanoscale Res. Lett.. 2012, 7, 337. DOI: 10.1186/1556-276X-7-337.

39. Fröhlich, E. The role of surface charge in cellular uptake and cytotoxicity of medical nanoparticles. Int. J. Nanomed. 2012, 7, 5577-5591. DOI: 10.2147/IJN.S36111.

40. Yildiz, I.; Shukla, S.; Steinmetz, N. F. Applications of viral nanoparticles in medicine Curr. Opin. Biotechnol. 2011, 22, 901-908. DOI: 10.1016/j.copbio.2011.04.020.

41. Chang, C. B.; Knobler, C. M.; Gelbart, W. M; Mason, T. G. Curvature dependence of viral protein structures on encapsidated nanoemulsion droplets. ACS Nano 2008, 2, 281-286. DOI: 10.1021/nn700385z. 
42. Park, J.; Estrada, A.; Sharp, K.; Sang, K.; Schwartz, J. A.; Smith, D.K.; Coleman, C.; Payne, J. D.; Korgel, B. A.; Dunn, A. K.; Tunnell, J. W. (2008). Two-photon-induced photoluminescence imaging of tumors using near-infrared excited gold nanoshells. Optics Express 2008, 16, 1590-1599.

43. Loredo-Tovias, M.; Duran-Meza, A. L.; Villagrana-Escareño, M. V.; Vega-Acosta, R.; ReynagaHernández, E.; Flores-Tandy, L. M.; Valdes-Resendiz, O. E.; Cadena-Nava, R. D.; Alvizo-Paez, E. R.; Ruiz-Garcia, J. Encapsidated ultrasmall nanolipospheres as novel nanocarriers for highly hydrophobic anticancer drugs. Nanoscale 2017, 9, 11625-11631. DOI: 10.1039/c7nr02118f.

44. Sivaraman, S. K.; Kumar, S.; Santhanam, V. Monodisperse sub-10nm gold nanoparticles by reversing the order of addition in Turkevich method - The role of chloroauric acid. J. Colloid Interface Sci. 2011, 361, 543-547. DOI: 10.1016/j.jcis.2011.06.015.

45. Duran-Meza, A. L.; Moreno-Gutierrez, D. S.; Ruiz-Robles, J. F.; Bañuelos-Frias, A.; Segovia-González, X. F.; Longoria-Hernandez, A. M.; Gomez, E.; Ruiz-Garcia, J. Synthesis and Characterization of Extremely Small Gold Nanoshells, and Comparison of Their Photothermal Conversion Capacity with Gold Nanorods. Nanoscale 2016, 8, 11091-11098. DOI: 10.1039/C6NR00027D.

46. Vongsavat, V.; Vittur, B. M.; Bryan, W. W.; Kim, J. H.; Lee, T. R. Ultrasmall hollow gold-silver nanoshells with extinctions strongly red-shifted to the near-infrared. ACS Appl. Mater. Interfaces 2011, 3, 3616-3624. DOI: 10.1021/am2008322.

47. Xu, D.; Mao, J.; He, Y.; Yeung, E. S. Size-tunable synthesis of high-quality gold nanorods under basic conditions by using $\mathrm{H}_{2} \mathrm{O}_{2}$ as the reducing agent. J. Mater. Chem. C 2014, 4989-4996. DOI:10.1039/C4TC00483C.

48. Michel, J. P.; Gingery, M.; Lavelle, L. Efficient purification of bromoviruses by ultrafiltration. J. Virol. Methods 2004, 122, 195-198. DOI: 10.1016/j.jviromet.2004.09.005.

49. Lavelle, L.; Michel, J. P.; Gingery, M. The disassembly, reassembly and stability of CCMV protein capsids. J. Virol. Methods 2007, 146, 311-316. DOI: 10.1016/j.jviromet.2007.07.020.

50. Burger, W.; Burge, M. J. Principles of Digital Image Processing; Springer-Verlag; London, England, 2009, pp. 1-24. ISBN: 978-1-84800-190-9

51. Melancon, M. P.; Lu, W.; Yang, Z.; Zhang, R.; Cheng, Z.; Elliot, A. M.; Stafford, J.; Olson, T.; Zhang, J. $\mathrm{Z}$.; Li, C. In vitro and in vivo targeting of hollow gold nanoshells directed at epidermal growth factor receptor for photothermal ablation therapy. Mol. Cancer Ther. 2008, 7, 1730-1739. DOI: 10.1158/15357163.MCT-08-0016.

Sample Availability: Samples of the compounds are not available from the authors. 